Aufsatz ZR

Prof. Dr. Christian Armbrüster*

\title{
Der Heiligabend - ein zivilrechtliches Kurzdrama in fünf Akten
}

https://doi.org/10.1515/jura-2020-2579

Der Beitrag zeigt - in der Darstellungsweise eines klassischen Dramas - einige klausurrelevante Themen des Schuld- und Sachenrechts auf, die allesamt auch jenseits des Heiligabends bedeutsam werden können. Der Reigen reicht von den gesetzlichen und vertraglichen Verschuldensmaßstäben über die werkvertragliche Gewährleistung und das Gleichbehandlungsrecht bis hin zu Fragen des Schenkungsrechts sowie des Eigentums- und Besitzübergangs. Ganz nebenbei erfährt man, wieso sich bei der Deliktshaftung in der Lebenswirklichkeit meist andere Beteiligte gegenüberstehen als in Klausuraufgaben. Kleine Ausflüge ins Feiertagsrecht in Prolog und Epilog runden den Beitrag ab.

\section{Prolog (Vorrede)}

Die Weihnachtstage markieren für die meisten Menschen hierzulande unverändert einen Höhepunkt im familiären Leben. Man könnte fast sagen, dass letztlich der gesamte Jahresverlauf auf den Advent und insbesondere auf den 24. Dezember zustrebt. Es ist daher durchaus bemerkenswert, dass dieser Tag gar kein gesetzlicher Feiertag ist. Bundeseinheitlich ist ohnehin nur der Tag der Deutschen Einheit als Feiertag festgelegt worden, und zwar in Art. 2 Abs. 2 Einigungsvertrag. ${ }^{1}$ Es besteht keine ausdrückliche Bundeskompetenz zur gesetzlichen Festlegung von bundeseinheitlichen Feiertagen. Sämtliche Bundesländer haben sich allerdings darauf verständigt, neun Feiertage durch ihre Sonn- und Feiertagsgesetze bundeseinheitlich

1 Einigungsvertrag vom 31. 8. 1990, BGBl. 1990 II S. 889.

Anmerkung: Der Beitrag ist hervorgegangen aus der Weihnachtsvorlesung, die der Autor am 12. 12. 2019 am Fachbereich Rechtswissenschaft der Freien Universität Berlin für die dortigen Studierenden gehalten hat.

*Kontaktperson: Christian Armbrüster, der Autor lehrt Privatrecht an der Freien Universität Berlin. zu schützen. ${ }^{2}$ Dazu zählen der erste und der zweite Weihnachtsfeiertag (25. und 26.12), aber eben nicht der Heiligabend, der in keinem Bundesland zum Feiertag gekürt wurde. ${ }^{3}$

Der Umstand, dass der Heiligabend kein gesetzlicher Feiertag ist, tut der festlichen Stimmung, die sich - wenn alles gut läuft - am Nachmittag oder frühen Abend bei vielen Familien einstellt, indes keinen Abbruch. Die Erwartungen sind jedes Jahr aufs Neue hoch. Dasselbe gilt für die Herausforderungen, die dieses Familienereignis für das menschliche Zusammensein mit sich bringt - und damit auch für die Juristerei.

Das Setting unseres Kurzdramas ist Folgendes: Ein gemietetes Reihenhaus am Stadtrand, Vater Thomas, Mutter Anna und der gemeinsame Sohn, der 5jährige Leo. Es ist Heiligabend, gegen 17 Uhr. Alle drei sind etwas aufgeregt, wenn auch aus unterschiedlichen Gründen. Leo hat in den vergangenen Wochen mehr als einmal erwähnt, dass ihn Drohnen faszinieren. Seine Eltern haben jeweils etwas einsilbig reagiert. Ganz sicher ist er sich daher nicht, ob die Botschaft angekommen ist. Thomas und Anna sind einander an sich wirklich sympathisch, sie haben aber bisweilen auch etwas am anderen auszusetzen. Er findet sie zu »öko«; sie hält ihn für zu träge. Deshalb weicht sie dem Thema Heirat bislang aus. Thomas spürt, dass es hier für ihn noch etwas zu tun gibt. Daher hat er - auch wenn ihm dabei mulmig ist - den festen Entschluss gefasst, dass er sich diesmal am Heiligabend richtig ins Zeug legen will: Schon vor Tagen hat er Anna mit der Ankündigung überrascht, dass er sich um das weihnachtliche Abendessen kümmern wird - es soll Ente geben -, dass er für Leo ein Unterhaltungsprogramm organisiert hat und dass Anna sich auf ein ganz besonderes Geschenk freuen könne. Anna ist gespannt.

2 https://www.bmi.bund.de/DE/themen/verfassung/staatlichesymbole/nationale-feiertage/nationale-feiertage-node.html (letzter Abruf am 22. 9. 2020).

3 S. etwa für Bayern: § 1 Feiertagsgesetz, BayRS 1131-3-I; für Berlin: § 1 Abs. 1 des Berliner Gesetzes über die Sonn- und Feiertage, GVBl. 1954, 615. 


\section{Akt: Das Weihnachtsmenü}

Thomas hat mit der Zubereitung des Weihnachtsmenüs begonnen. Er kämpft gerade mit der Plastikverpackung der tiefgefrorenen $»$ Duck Chunks « aus chinesischer Produktion, die er im Supermarkt erstanden hat. Auf dem Herd der Einbauküche steht schon der Topf mit heißem Frittieröl bereit. Da klingelt es an der Haustür. Ein Nachbar braucht Starthilfe für sein Kfz. Thomas geht in die Garage, um sein eigenes Auto zu holen, und fährt damit zum Nachbarn. Als er nach einer Viertelstunde zurückkehrt, schlagen schon Rauschschwaden aus dem Küchenfenster.

Als Thomas dem Gebäudeversicherer seines Vermieters den Schaden meldet, nimmt der Bearbeiter die Schadensmeldung gleichmütig entgegen. Er kennt das schon: Der unbeaufsichtigt auf dem eingeschalteten Herd stehen gelassene Topf mit heißem Öl ist für ihn ein »Klassiker«, nicht nur zur Weihnachtszeit. ${ }^{4}$ Dabei sollte es doch allgemein bekannt sein, dass heißes Öl sich selbst entzünden kann. Thomas ist etwas kleinlaut. Er offenbart dem Sachbearbeiter, dass er seit seiner Studienzeit nicht mehr an einem Herd stand, und dass ihm damals bei der Zubereitung seines Standardessens (Spaghetti mit Fertigsauce) dergleichen nie passiert sei. Der Sachbearbeiter erklärt geduldig, dass Nudelwasser andere physikalische Eigenschaften hat als Frittieröl. Im Stillen denkt er sich, dass es ihm lieber gewesen wäre, wenn ein erfahrener Koch den Brand verursacht hätte. Wieso?

Die Frage lässt aufhorchen. Im deutschen Zivilrecht gilt grundsätzlich ein einheitlicher Verschuldensmaßstab. Dies bedeutet, dass es für die Haftung eines Schädigers etwa nach $\S 823$ Abs. 1 BGB keinen Unterschied macht, ob er den Schaden mit leichtester Fahrlässigkeit (culpa levissima) oder ganz im Gegenteil mit Absicht (dolus directus 1. Grades) verursacht hat. In jedem Fall hat er für den vollen Schaden aufzukommen. Dies hängt mit der Ausgleichsfunktion des Haftungsrechts zusammen: Der Schädiger soll dann, wenn er schuldhaft gehandelt hat, dem Geschädigten den Schaden zu ersetzen haben. ${ }^{5}$ Hingegen geht es nicht darum, sein Verhalten mit einer Sanktion zu ahnden, was eine Abstufung der Rechtsfolgen gebieten würde, wie sie im Straf- und Ordnungswidrigkeitenrecht erfolgt. Hinsichtlich der weiteren Funktion des Haftungsrechts, präventiv eine verhaltenssteuernde Wirkung zu erzielen, ${ }^{6}$ liegt der einheitliche Verschuldensmaßstab

4 Es gibt zahlreiche Gerichtsentscheidungen zu der Thematik; s. nur BGH VersR 2011, 916; OLG Köln VersR 2002, 311; LG Dortmund r+s 2012, 27; LG Göttingen r+s 2015, 611; für Österreich OGH VersR 2005, 815. 5 MünchKomm-BGB/Wagner, 8. Aufl. 2020, Vor $\S 823$ Rn. 43 f. 6 MünchKomm-BGB/Wagner, 8. Aufl. 2020, Vor § 823 Rn. $45 \mathrm{ff}$. nicht auf der Hand; schließlich ist die Verhaltenssteuerung etwa bei grober Fahrlässigkeit bedeutsamer als bei einer leichten Unachtsamkeit im Sinne eines Augenblicksversagens. Aus Sicht des Geschädigten ist es indessen gleichgültig, mit welchem Verschuldensgrad der Schaden verursacht worden ist. Darin liegt der entscheidende Grund für den einheitlichen Maßstab. Nur dann, wenn den Schädiger keinerlei Verschuldensvorwurf trifft, erscheint es grundsätzlich (vorbehaltlich der Fälle der Gefährdungshaftung ${ }^{7}$ ) angemessen, dem Geschädigten einen Ausgleichsanspruch zu verwehren. Bei dem Schaden handelt es sich dann um eine Ausprägung des sog. allgemeinen Lebensrisikos.

Wieso also sollte es für den Versicherer einen Unterschied machen, wie erfahren Thomas beim Kochen ist? Der Grund liegt darin, dass der einheitliche Verschuldensmaßstab zwar im Deliktsrecht regelmäßig uneingeschränkt gilt, im Vertragsrecht hingegen häufig modifiziert wird. Dass solche Modifizierungen sowohl in Richtung einer strengeren als auch einer milderen Haftung in gewissen Grenzen rechtlich zulässig sind, folgt grundsätzlich aus § $276 \mathrm{Abs} .1$ S. 1 Halbs. 2 BGB. In der Praxis sind Haftungsbeschränkungen häufig in AGB anzutreffen. Insoweit werden die Grenzen der Gestaltungsfreiheit insbesondere durch $\S 309$ Nr. 7 lit. b BGB markiert. Demnach sind Klauseln unwirksam, mit denen die Haftung des Verwenders für eigene grobe Fahrlässigkeit oder für vorsätzliches oder grob fahrlässiges Verhalten seiner Erfüllungsgehilfen ausgeschlossen werden soll. Der auf den ersten Blick verblüffende (und daher gern in mündlichen Prüfungen thematisierte) Umstand, dass in diesem Klauselverbot eigenes vorsätzliches Verhalten des Verwenders nicht erwähnt wird, ist darauf zurückzuführen, dass insoweit ein vertraglicher Haftungsausschluss bereits nach der allgemeinen Vorschrift des $\S 276$ Abs. 3 BGB unzulässig ist, also auch dann, wenn er nicht in AGB i.S.v. § 305 BGB, sondern in einer Individualabrede enthalten ist.

Der Schadensachbearbeiter hat hier Vertragsrecht anzuwenden, nämlich konkret das im Gebäudeversicherungsvertrag abgegebene Leistungsversprechen des Versicherers. Das Privatversicherungsrecht ist in weiten Teilen besonderes AGB-Recht. Versicherungsverträge sind nämlich anders als eine physisch wahrnehmbare Leistung wie etwa ein Laptop oder Fahrrad als Kaufsache reine Rechtsprodukte; die vom Versicherer im Gegenzug zur Prämienzahlung versprochene Leistung folgt hier allein aus den im Vertrag getroffenen Abreden. Im Kern geht es

$7 \mathrm{Zu}$ ihr s. Medicus/Petersen, Bürgerliches Recht, 27. Aufl. 2019, Rn. $631 \mathrm{ff}$. 
darum, dass bei Eintritt bestimmter vorab definierter Umstände (sog. Versicherungsfall, z. B. Brandschaden im versicherten Gebäude) eine bestimmte Entschädigung zu leisten ist. Dabei kann außer den Voraussetzungen auch die Höhe dieser Entschädigung wesentlich von den Regeln abweichen, die für deliktische Schadensersatzansprüche nach den $\S \S 249 \mathrm{ff}$. BGB gelten. So ist es beispielsweise in der Gebäudeversicherung üblich, dass der Versicherer bei einer Zerstörung des Gebäudes nicht nur dessen Zeitwert, sondern die Kosten des Wiederaufbaus trägt, und zwar ohne Abzug "neu für alt «\$, wenn der Versicherungsnehmer diesen Wiederaufbau innerhalb von drei Jahren sicherstellt. Diese Regel soll dem tatsächlichen Finanzbedarf des Eigentümers Rechnung tragen und zugleich verhindern, dass ein Anreiz geschaffen wird, den Versicherungsfall zwecks Geldbeschaffung heimlich selbst herbeizuführen. Weil die Festlegung dieser Punkte und weiterer Themen wie etwa der vom Versicherungsnehmer zu befolgenden Verhaltensregeln (sog. Obliegenheiten) einer verbalen Umschreibung bedarf, sind im Versicherungssektor schon sehr frühzeitig AGB entwickelt worden, lange bevor es sie etwa für Kaufverträge gab. Dementsprechend spielen Allgemeine Versicherungsbedingungen (AVB) für die Entwicklung des AGB-Rechts bereits in der Rechtsprechung des Reichsgerichts eine große Rolle.

Doch kommen wir zurück zur Ausgangsfrage. Unproblematisch ist hier, dass der Versicherer für den Brandschaden an den Gebäudebestandteilen gegenüber dem Vermieter als dem Eigentümer des Reihenhauses aufkommen muss (am Rande: nicht jeder Vermieter einer Sache ist zugleich deren Eigentümer; fehlen abweichende Sachverhaltsangaben, ist aber gemäß der Lebenswirklichkeit von einer Identität auszugehen). Ein Problem kann sich hier nur dann ergeben, wenn bei dem Brand auch die Einbauküche zu Schaden gekommen ist, wovon bei lebensnaher Betrachtung auszugehen ist. In diesem Fall kommt es darauf an, ob die Küche als Gebäudebestandteil anzusehen ist. Manche Bedingungswerke zur Gebäudeversicherung treffen dazu eine ausdrückliche Bestimmung. Fehlt eine Regelung, so muss die Frage anhand der allgemeinen Vorschriften beantwortet werden, die das Gesetz für Grundstücksbestandteile aufstellt. ${ }^{9}$

Erfüllt eine Einbauküche im Einzelfall die Voraussetzungen der Legaldefinition von wesentlichen Bestandteilen in $\S 93$ BGB, so folgt daraus, dass sie zum Gebäude gehört. Dies setzt voraus, dass die Einbauküche dem Ge-

8 S. zu diesem Abzug BGHZ 102, 322 = NJW 1988, 1835, 1836. 9 Instruktiv zum Folgenden BeckOGK BGB/Mössner, Stand 1. 4. 2020, $\S 94$ Rn. 26.4, 28.1. bäude besonders angepasst ist, so dass sie nicht davon getrennt werden kann, ohne dass sie zerstört oder in ihrem Wesen verändert wird. Davon ist etwa dann auszugehen, wenn die Einbauküche schon im Bauplan auf die räumliche Gestaltung abgestimmt oder wenn sie in den Küchenraum eingemauert worden ist. Hingegen reicht es nicht, wenn eine aus seriell hergestellten Bauteilen (Modulen) bestehende Einbauküche lediglich durch Blenden oder einen individuellen Zuschnitt der Arbeitsplatte an den Raum angepasst wurde. ${ }^{10}$

Sind die strengen Anforderungen des $\S 93$ BGB nicht erfüllt, so richtet sich die Beurteilung nach $\S 94$ Abs. 2 BGB. Diese Vorschrift erklärt die zur Herstellung des Gebäudes eingefügten Sachen zu wesentlichen Bestandteilen des Gebäudes. Maßgeblich für die Beurteilung, ob es sich bei der Einbauküche um eine zur Herstellung des Gebäudes eingefügte Sache handelt, ist die Verkehrsanschauung. Nach ihr richtet sich, ob erst die Einfügung der Einbauküche dem Gebäude ein bestimmtes Gepräge gibt. Diese Frage ist innerhalb von Deutschland in der Vergangenheit von vielen Gerichten uneinheitlich beurteilt worden. So gibt es Judikatur, die für Norddeutschland eine Verkehrsanschauung bejaht, der zufolge ein Wohngebäude bzw. eine Wohnung erst mit der Einfügung einer Einbauküche als fertiggestellt anzusehen ist. Für viele andere Regionen ist dies hingegen verneint worden, und auch für Norddeutschland weisen jüngere Gerichtsentscheidungen in diese Richtung. Gerade bei Normen wie $\S 94$ Abs. 2 BGB, die nicht zuletzt für das von einem Interesse an Rechtsklarheit geprägte Sachenrecht praktisch sehr bedeutsam sind, ist eine regionale Differenzierung nach einer (empirisch regelmäßig nicht belegten) Verkehrsanschauung problematisch. Hinzu kommt, dass angesichts der mittlerweile hohen Mobilität in der Bevölkerung traditionelle regionale Vorstellungen davon, was zur Herstellung eines Gebäudes erforderlich ist, entscheidend an Bedeutung verloren haben.

Daher erscheint es richtig, generell davon auszugehen, dass Einbauküchen nicht gem. § 94 Abs. 2 BGB als wesentliche Gebäudebestandteile anzusehen sind. ${ }^{11}$ Es handelt sich vielmehr um Zubehör i.S.v. § 97 Abs. 1 BGB. Wiederum anders ist die Lage zu beurteilen, wenn die Einbauküche nicht mitvermietet (oder dem Mieter rein tatsächlich verfügbar gemacht, ohne als Teil der den Gewährleistungsregeln unterliegenden Mietsache zu gelten), sondern vom Mieter eingebracht worden wäre. In diesem

10 OLG Koblenz NJW-RR 2017, 838 Rn. 23.

11 OLG Nürnberg NJW-RR 2002, 1485, 1486; BeckOGK BGB/Mössner, Stand 1. 4. 2020, § 94 Rn. 26.4. 
Fall würde es sich um einen Scheinbestandteil i.S.v. § 95 Abs. 2 BGB handeln. ${ }^{12}$ Zwischenfazit: Es hängt von den tatsächlichen Gegebenheiten ab, ob der Gebäudeversicherer des Vermieters auch hinsichtlich der Einbauküche leistungspflichtig ist oder nicht. In jedem Fall besteht eine Eintrittspflicht hinsichtlich der durch den Brand am Gebäude selbst entstandenen Schäden, etwa an Fenstern, Böden oder Elektroleitungen.

Doch nun wieder zurück zum Fall! Auf den ersten Blick hat Thomas mit dem Schaden, dessen Ersatz sein Vermieter vom Gebäudeversicherer verlangen kann, gar nichts $\mathrm{zu}$ tun. Schließlich wird der Vermieter dadurch schadlos gestellt. Allerdings ist die Rechtslage komplizierter: Die Leistung des Versicherers an den geschädigten Eigentümer hat nämlich für den Schadensverursacher keine haftungsbefreiende Wirkung. Zwar handelt es sich bei dem Schadensersatzanspruch aus $\S 823$ Abs. 1 BGB um eine nicht persönlich zu erbringende Leistung. Daher kann nach $\S 267$ Abs. 1 S. 1 BGB grundsätzlich auch ein Dritter die Leistung bewirken (Ausnahme: Abs. 2). Indessen hat der Versicherer dann, wenn er an den Geschädigten zahlt, nicht den Willen, damit die Schuld des Haftpflichtigen zu tilgen. Vielmehr erfolgt die Zahlung aufgrund des Leistungsversprechens, das der Versicherer im Versicherungsvertrag gegenüber dem Geschädigten abgegeben hat. Der Versicherer kann in dem Umfang, in dem er dem Eigentümer den Schaden tatsächlich ersetzt, gegen den Schädiger Rückgriff nehmen. Dies folgt aus $\S 86$ Abs. 1 VVG, der einen gesetzlichen Forderungsübergang (Legalzession) anordnet; für Sozialversicherungsträger sieht § 116 SGB X unter ähnlichen Voraussetzungen - es kommt dort nicht auf den tatsächlichen Ersatz an; vielmehr genügt die Verpflichtung dazu - gleichfalls eine Legalzession vor.

Hier hat sich Thomas wegen der durch den Brand verursachten Eigentumsverletzung gegenüber dem Vermieter haftbar gemacht, und zwar aus Mietvertragsrecht gem. $\S \S 280$ Abs. 1, 241 Abs. 2 BGB (mit der Haftungsmilderung hinsichtlich Abnutzung durch vertragsgemäßen Gebrauch gem. § 538 BGB, der hier überschritten wurde) und aus Delikt gem. § 823 Abs. 1 BGB. Allerdings besteht im Ergebnis Einigkeit darüber, dass Thomas für einen Schaden, den er seinem Vermieter leicht fahrlässig zugefügt hat, nicht soll aufkommen müssen. Zur Begründung wird darauf verwiesen, dass er durch die Mietzahlung die Gebäudeversicherungsprämie finanziert, sei es in Gestalt einer eigenen Kostenposition bei den umlagefähigen Nebenkosten oder - im Fall einer Bruttomiete - zumindest kalkulatorisch, da der

12 Staudinger/Stieper, BGB, 2017, § 95 Rn. 16.
Vermieter solche Kosten bei der Festlegung der Miete berücksichtigt.

Früher hat der BGH den Schutz des Mieters rechtstechnisch teils dadurch zu erreichen versucht, dass er mittels ergänzender Auslegung eine stillschweigende Haftungsbegrenzung auf grobe Fahrlässigkeit in den Mietvertrag hineinlas. ${ }^{13}$ Diese Rechtsprechung ist zu Recht auf Kritik gestoßen, da sie sich vom mutmaßlichen Willen des Vermieters entfernt, für den kein Anlass besteht, sich etwa dann, wenn ihm aufgrund einer Inanspruchnahme des Versicherers Nachteile (Kündigung des Versicherungsvertrags, Prämienerhöhung) drohen oder wenn der Versicherer aus irgendeinem Grund leistungsfrei ist, ohne Weiteres seiner vertraglichen und deliktischen Schadensersatzansprüche gegen den Mieter zu begeben. Mittlerweile verwirklicht der BGH den Schutz des Mieters auf der versicherungsrechtlichen Ebene: In den Versicherungsvertrag wird ein stillschweigender Regressverzicht des Versicherers für solche Schäden hineingelesen, die der Mieter des Versicherungsnehmers leicht fahrlässig verursacht hat. ${ }^{14}$ Diese ergänzende Auslegung entspricht dem mutmaßlichen Willen beider Parteien des Versicherungsvertrags: Der Vermieter (und Versicherungsnehmer) hat ein dem Versicherer erkennbares Interesse daran, dass das Mietverhältnis nicht durch einen Regress des Versicherers belastet wird.

Dem Versicherer ist dieser Regressverzicht zumutbar, da infolge der Vermietung der Mieter als Nutzer (und damit als potentiell Haftpflichtiger) an die Stelle des Vermieters getreten ist. Hätte der Vermieter das ihm gehörende Reihenhaus selbst bewohnt, so wäre ihm der Versicherer bei eigener leicht fahrlässiger Schadensverursachung stets in vollem Umfang leistungspflichtig. Dies folgt aus der (dispositiven) Vorschrift des § 81 VVG, wonach der Anspruch gegen den Versicherer bei Vorsatz entfällt und er bei grober Fahrlässigkeit gekürzt werden kann, bei leichter Fahrlässigkeit hingegen unberührt bleibt. Damit typisiert der Gesetzgeber das mutmaßliche Interesse der Parteien: Das Leistungsversprechen des Versicherers soll den Versicherungsnehmer auch dann schützen, wenn dieser den Versicherungsfall selbst leicht schuldhaft herbeigeführt hat. Damit wird dem Umstand Rechnung getragen, dass die Prämienzahlung auch ein eigenes fehlerhaftes Verhalten absichern soll, das in der Lebenswirklichkeit selbst Personen unterlaufen kann, die sich normalerweise sorgfältig verhalten.

13 BGHZ 131, 288, 292 = NJW 1996, 715.

14 Grundlegend BGHZ 145, 393, 397 ff. = NJW 2001, 1353; näher Armbrüster, Privatversicherungsrecht, 2. Aufl. 2019, Rn. $1409 \mathrm{ff}$. 
Was bedeutet all dies letztlich für Thomas? Indem er den eingeschalteten Herd mit dem Öl für eine Viertelstunde unbeaufsichtigt ließ, hat er diejenige Vorsicht außer Acht gelassen, die jedermann unmittelbar einleuchten muss. Von einem durchschnittlichen Angehörigen des maßgeblichen Verkehrskreises (hier: Erwachsener ohne geistige Einschränkungen) kann nämlich ein verantwortungsvoller Umgang mit Kochplatten und mit siedendem Öl erwartet werden. Das Verhalten von Thomas ist damit als objektiv grob fahrlässig einzustufen. Der BGH verlangt allerdings im hier interessierenden Kontext darüber hinaus für grobe Fahrlässigkeit, dass das Verhalten auch in subjektiver Hinsicht schlechthin unentschuldbar war. Diese Einschränkung kann für Thomas der Rettungsanker sein: In einem ganz vergleichbaren Fall meinte der BGH, es sei zugunsten des (immerhin 33jährigen) Mieters zu berücksichtigen, dass dieser »erst seit relativ kurzer Zeit eigene Erfahrungen mit der Essenszubereitung gesammelt $\aleph^{15}$ hatte. Hält man Thomas seine mangelnde Kocherfahrung zugute, so entgeht er dem Regress des Gebäudeversicherers. Ein routinierter Koch hätte sich hingegen schwerlich darauf berufen können, dass das Verhalten subjektiv nicht schlechthin unentschuldbar war; der BGH gestattet dem Versicherer in solchen Fällen den vollen Regress. ${ }^{16}$

Die obigen Ausführungen zum Versicherungsrecht mögen recht speziell erscheinen. Allerdings verdeutlichen sie einen wichtigen Unterschied zwischen dem »wahren Leben « und der Welt der Klausuraufgaben, die ja eigentlich den Anspruch hat lebensnah zu sein: In der Wirklichkeit erfolgt die Inanspruchnahme eines Deliktsschuldners sehr häufig nicht durch den Geschädigten, sondern durch einen Versicherer, der diesem den Schaden aufgrund eines Versicherungsvertrags ersetzt hat. Besonders deutlich wird dies bei Personenschäden: Wer durch einen anderen verletzt wird, überlässt die Auswahl des Arztes in der Regel nicht dem Schädiger (vgl. § 249 Abs. 1 BGB), und er verlangt von diesem auch nicht den für die Heilbehandlung erforderlichen Geldbetrag (vgl. § 249 Abs. 2 S. 1 BGB). Vielmehr übernimmt die Kostentragung der gesetzliche oder private Krankenversicherer des Geschädigten, der seinerseits den Schadenshergang ermittelt und ggf. den Schädiger in Regress nimmt. Verfügt der Schädiger seinerseits über Haftpflichtversicherungsschutz, so stehen sich im Prozess anstelle von Schädiger und Geschädigtem zwei Versicherer gegenüber. Umsichtige Klausuraufgabenstel-

15 BGH VersR 2011, 916 Rn. 13 (dort ging es um tiefgefrorene Kartoffelröllchen).

16 BGH NJW-RR 2017, 22 Rn. 14 ff.; für Quotelung dagegen zu Recht etwa Looschelders, r+s 2015, 581, 584. ler bringen daher gern bei den Bearbeiterhinweisen die Aussage an, dass auf versicherungsrechtliche Fragen nicht einzugehen ist (auch wenn in manchen Gesetzesausgaben sogar die $\S \S 81$ VVG, 116 SGB X eigens als Fußnote zu $\S 823$ BGB abgedruckt sind). Für Studierende ist dies erfreulich; für die spätere Praxis sollte man allerdings die wahren Abwicklungsverhältnisse kennen.

\section{Akt: Der Adventskranz}

Nachdem Thomas sich für seine Kochaktion in die Küche zurückgezogen hat, macht Anna es sich im Wohnzimmer gemütlich. Sie will einer alten Familientradition entsprechend die Kerzen des Adventskranzes "ausbrennen« lassen. Da klingelt es. Diesmal ist es das Telefon im Flur. Am Apparat ist, wie jedes Jahr an Heiligabend um diese Uhrzeit, Onkel Leopold aus Altötting. Anna ist genervt. Sie zeigt ihren Unwillen aber nicht; immerhin ist Onkel Leopold Leos Patenonkel und sehr vermögend, und sie muss doch an die Zukunft des Kleinen denken! Während Anna noch im Flur sitzt und mit Onkel Leopold plaudert, vernimmt sie ein immer lauter werdendes Knistern aus dem Wohnzimmer.

Auch diesmal bleibt der Schadenssachbearbeiter des Gebäudeversicherers zunächst gelassen. Der brennende Adventskranz ist schließlich in der gesamten Adventszeit und noch bis in den Januar hinein - wenn die Zweige immer trockener werden - ein saisonaler »Klassiker«. Als Anna berichtet, dass der Adventskranz aus Stroh war, horcht der Sachbearbeiter allerdings auf. Ist das nicht grobe Fahrlässigkeit, so dass der Gebäudeversicherer gegen Anna Rückgriff nehmen kann?

Die bereits zitierte Formel des BGH, wonach grob fahrlässig nur handelt, wer sich auch in subjektiver Hinsicht schlechthin unentschuldbar verhält, erfordert stets eine Einzelfallbetrachtung. Während für den rein objektiven Fahrlässigkeitsmaßstab des § 276 BGB abstrakte Anforderungen formuliert werden können (z.B. das Führen eines Kfz im Zustand absoluter Fahruntauglichkeit oder eben das unbewachte Brennenlassen von Kerzen), liegt hinsichtlich der subjektiven Komponente jeder Fall anders. Hier mag Anna geltend machen, dass sie als überzeugte Pflanzenfreundin kein frisches Grün seiner Lebensader berauben wolle und deshalb das (leicht entzündliche und damit die Brandgefahr gegenüber einem konventionellen Adventskranz erhöhende) Stroh vorgezogen habe. Ob dieses Vorbringen geeignet wäre, sie vom Vorwurf des auch subjektiv schweren Verschuldens zu entlasten, ist ebenso zweifelhaft wie dies hinsichtlich des Umstands gilt, dass sie das Telefonat deshalb nicht abgekürzt hat, 
weil sie kein Risiko hinsichtlich des erhofften künftigen Erbes für Leo setzen wollte.

Grundsätzlich legt die Rechtsprechung freilich einen schädigerfreundlichen Maßstab an, wenn es um die Frage geht, ob das Verhalten auch subjektiv unentschuldbar war. Ein anschauliches Beispiel, das zugleich mögliche weitere Turbulenzen eines Weihnachtsfests aufzeigt, bietet ein Urteil des OLG Oldenburg. Sachverhalt und rechtliche Würdigung sind im Leitsatz des Urteils so anschaulich und kompakt wiedergegeben, dass dieser hier wörtlich zitiert werden soll: »Das Brennenlassen von Kerzen in unbeaufsichtigtem Zustand mit der Folge eines Zimmerbrands erfüllt den Tatbestand der grob fahrlässigen Herbeiführung des Versicherungsfalls dann nicht, wenn die (mitversicherte) Ehefrau des Versicherungsnehmers sich der Gefahr durchaus bewusst ist und die Kerzen deshalb vor Verlassen der Wohnung löschen will, jedoch nicht entsprechend ihrer ursprünglichen Absicht handelt, weil es $\mathrm{zu}$ einer heftigen Auseinandersetzung mit einem an sich folgsamen zehnjährigen Sohn kommt, der sich weigert, die Wohnung für einen geplanten Verwandtenbesuch $\mathrm{zu}$ verlassen, während zwei weitere vier- und achtjährige Kinder schon zu dem vor dem Haus parkenden Pkw laufen, in dem der Versicherungsnehmer auf die Familie wartet und hupt. $\ll^{17}$

Vergleichbar ist etwa die Situation, dass eine Mieterin zu Weihnachten nicht an die brennenden Kerzen denkt, weil sie mit ihrem quengelnden Kleinkind den neuen Puppenwagen vor dem Haus kurz ausprobiert. ${ }^{18}$ Der $»$ Klassiker« ist allerdings tatsächlich die Ablenkung durch ein Telefonat, ${ }^{19}$ so wie es Anna widerfahren ist.

\section{Akt: Der Weihnachtsmann}

Es klingelt schon wieder. Diesmal ist es der Weihnachtsmann. Jetzt kommt also das angekündigte Unterhaltungsprogramm, denkt sich Anna. Thomas hatte zuvor die im Internet beworbenen Weihnachtsmann-Kostüme gesehen, zugleich aber eine Anzeige der Agentur »Call-a-Weihnachtsmann « entdeckt, die den kompletten Auftritt eines Weihnachtsmanns mit kindgerechter Lob- und Mahnrede sowie Überreichung der zuvor vom Kunden vor dem Haus deponierten Geschenke anbietet. Das schien Thomas die komfortablere Variante als ein eigener Auftritt zu sein. Er hat daher bei der Agentur den Auftritt gebucht. Als Leo im

17 OLG Oldenburg NJW-RR 2000, 480.

18 OLG Düsseldorf NJW-RR 1998, 1636.

19 S. etwa OLG Hamm r+s 1989, 334.
Wohnzimmer den Weihnachtsmann erblickt, bricht er nach einer Schrecksekunde in Tränen aus. Anna bemerkt an dem Weihnachtsmann zunächst nichts Besonderes. Erst als dieser den Arm mit dem mahnenden Zeigefinger in die Höhe streckt, um zu seiner Ansprache für Leo anzusetzen, fällt Annas Blick zufällig auf die schlanken Hände des Weihnachtsmanns. Da fällt es ihr schlagartig auf: Der Weihnachtsmann ist in Wahrheit eine Weihnachtsfrau! Sofort unterbricht Anna die Ansprache und stellt Thomas zur Rede. Dieser ist nun selbst ganz überrascht. Er beteuert wahrheitsgemäß, dass er einen Weihnachtsmann-Auftritt bestellt hat.

Ist eine Weihnachtsfrau ein mangelhafter Weihnachtsmann? Die Frage wird rechtlich bedeutsam, wenn es darum geht, ob Thomas von der Agentur als seiner Vertragspartnerin die $65 €$ zurückverlangen kann, die bereits von seinem Kreditkartenkonto abgebucht worden sind. In Betracht kommt ein Rücktritt nach $\S \S 633,634$ Nr. 3, 636, 323 Abs. 1, 326 Abs. 5, 275 Abs. 1 BGB. Voraussetzung ist zunächst, dass ein Werkvertrag vorliegt. Vereinbart war hier ein Auftritt als Weihnachtsmann. Bei einem solchen schauspielerischen Einsatz kann es sich um einen Werkoder Dienstvertrag handeln. Diese beiden Vertragstypen unterscheiden sich dadurch, dass der Werkvertrag auf die Herstellung eines bestimmten Werks gerichtet und mithin erfolgsbezogen ist, der Dienstvertrag hingegen tätigkeitsbezogen. Einen Anhaltspunkt für die Abgrenzung bietet die Vergütung: Ist diese pauschal vereinbart, spricht das für einen Werkvertrag; eine stundenweise Bezahlung deutet auf einen Dienstvertrag hin. Hier ist angesichts des vereinbarten Pauschalbetrags und der zu erbringenden Leistungen, die zusammengenommen den Erfolg darstellen, davon auszugehen, dass ein Werkvertrag vorliegt.

Das erstellte Werk muss einen Sachmangel aufweisen. Dies ist nach $\S 633$ Abs. 1, 2 BGB dann der Fall, wenn sich das Werk für die vereinbarte, hilfsweise für die vertraglich vorausgesetzte oder für die gewöhnliche Verwendung nicht eignet. Thomas verweist gegenüber »Call-a-Weihnachtsmann« darauf, dass Leo beim Anblick der Weihnachtsfrau in Tränen ausgebrochen ist. (Dabei verschweigt er freilich, dass Leo nur deshalb traurig war, weil er blitzartig erfasst hat, dass das Päckchen der Weihnachtsfrau viel zu klein für die Drohne war, die er sich so sehnlich gewünscht hatte.) Die Agentur entgegnet, dass Weihnachtsfrauen statistisch belegbar viel einfühlsamer mit den Kindern umzugehen verstünden als ihre männlichen Kollegen. Im Übrigen sei es diskriminierend, wenn Weihnachtsfrauen als mangelhafte Weihnachtsmänner behandelt würden.

Sofern der Vertrag keine Präzisierung der Leistung enthält, kommt es für die Frage der Mangelhaftigkeit gem. $\S 633$ Abs. 2 S. 1 Nr. 1 BGB auf die nach dem Vertrag vo- 
rausgesetzte Eignung an. Wer den Auftritt eines Weihnachtsmanns bucht, verbindet damit die Erwartung, dass eine Person mit dem üblichen Weihnachtsmann-Kostüm auftritt, zu dem auch ein längerer weißer Bart gehört. Solange für den Adressatenkreis des Auftritts - hier: Kinder im Alter von Leo - dieses äußere Erscheinungsbild gewahrt ist, kann es nicht darauf ankommen, welches Geschlecht die unter der Kostümierung verborgene Person hat. Auch aus der Stimmlage kann der Besteller nichts für sich herleiten, sofern diese nicht völlig vom üblichen Auftreten eines Weihnachtsmannes abweicht (oder die Person z. B. in einer für die Kinder nicht verständlichen Sprache spricht). Hier ist Anna erst durch einen Blick auf die Hände aufgefallen, dass sich unter dem Kostüm eine Frau verbarg; Leo als der Adressat des Auftritts hat dies gar nicht bemerkt. Mithin ist die von der Agentur erbrachte Werkleistung nicht mangelhaft. Thomas hat daher schlechte Karten, wenn er den Werklohn zurückfordert.

Variante 1: Anders kann die Lage zu beurteilen sein, wenn die Weihnachtsfrau sich von vornherein deutlich als Frau zu erkennen gibt und z. B. nicht den üblichen weißen Bart trägt. An der Bedeutung der oben genannten Erwartung, wie ein Weihnachtsmann auszusehen hat, ändert sich auch dadurch nichts, dass der Weihnachtsmann anders als der Nikolaus - der seinen Auftritt bekanntlich schon am 6. Dezember hat - nicht eine religiös geprägte historische Figur repräsentieren soll, sondern erst seit 1931 von einem US-amerikanischen Softdrinkhersteller durch Werbeillustrationen einem breiten Publikum bekannt geworden ist. Auch der Begriff "Weihnachtsmann« in der Firma der Agentur (merke: Firma ist nach $\S 17$ Abs. 1 HGB der Name, unter dem ein Kaufmann seine Geschäfte betreibt, und nicht der unter einer Firma handelnde Unternehmensträger) deutet darauf hin, dass eine männliche Verkleidung erforderlich ist. Daher ist beim Auftritt einer für die Zielgruppe der Kinder als solche erkennbaren Frau davon auszugehen, dass sich das Werk für die vertraglich vorausgesetzte oder zumindest für die gewöhnliche Verwendung nicht eignet und mithin mangelhaft ist. Der von Thomas bestellte Auftritt als Weihnachtsmann am Heiligabend kann zwar durch eine andere Person noch vorgenommen werden, aber hier nicht mehr den vertragsmäßigen Erfolg erreichen. Er ist daher als absolutes Fixgeschäft einzuordnen. ${ }^{20}$ Mithin ist infolge Zeitablaufs Unmöglichkeit i.S.v. § 275 Abs. 1 BGB eingetreten. Thomas kann demnach in der Variante gem. §§ 326 Abs. 5, 323

20 S. allg. Medicus/Petersen, Bürgerliches Recht, 27. Aufl. 2019, Rn. 160.
Abs. 1 BGB ohne Fristsetzung von dem mit der Agentur geschlossenen Vertrag zurücktreten.

Die Agentur macht allerdings geltend, es sei diskriminierend, wenn Weihnachtsfrauen als mangelhafte Weihnachtsmänner behandelt würden. Damit ist die Frage aufgeworfen, ob der Rücktritt gegen eines der Diskriminierungsverbote des AGG verstößt. In Rede steht eine unmittelbare Benachteiligung wegen des Geschlechts. Beim Geschlecht handelt es sich um ein geschütztes Merkmal i.S.v. § 1 AGG. In Betracht kommt hier ein Verstoß gegen das geschlechtsbezogene Benachteiligungsverbot gem. § 19 Abs. 1 Nr. 1 AGG. Dieses Verbot erfasst nicht allein Benachteiligungen bei der Begründung eines Vertragsverhältnisses, indem z.B. Mietinteressenten mit einer bestimmten ethnischen Herkunft oder $"$ Rasse $«^{21}$ der Vertragsschluss verweigert wird. ${ }^{22}$ Der Schutz vor Diskriminierungen erstreckt sich vielmehr auch auf die Durchführung und die Beendigung zivilrechtlicher Schuldverhältnisse.

Hier kommt in Betracht, dass die Weihnachtsfrau bei der Beendigung des Werkvertrags wegen ihres Geschlechts benachteiligt wurde, indem Thomas von dem mit der Agentur geschlossenen Vertrag mit der Begründung zurücktrat, dass die Erfüllung durch eine Frau einen Mangel bedeute. Ein Verstoß gegen § 19 Abs. 1 Nr. 1 AGG liegt hier allerdings aus mehreren Gründen nicht auf der Hand. So führt der Rücktritt nicht zur Beendigung des Schuldverhältnisses, sondern gem. § 346 BGB zu dessen Umwandlung in ein Rückgewährschuldverhältnis. Diesen Einwand wird man bei einer am Schutzzweck des Gesetzes orientierten Auslegung überwinden können. ${ }^{23}$ Dasselbe gilt für den Umstand, dass die Weihnachtsfrau als Mitarbeiterin der Agentur gar nicht Vertragspartnerin von Thomas als demjenigen ist, bei dem ein diskriminierendes Verhalten in Rede steht. Schwerer wiegt der Einwand, dass Thomas hier nicht der Anbieter, sondern Nachfrager der vereinbarten Werkleistung ist. Die Verpflichtung zur Gleichbehandlung trifft aber auch bei der Beendigung allein den ursprünglichen Anbieter. ${ }^{24}$ Das AGG soll verhindern, dass die Anbieter von Beschäftigung, Waren oder Dienstleistungen einem bestimmten Personenkreis wegen eines der geschützten Merkmale den Zugang zu Schuld-

$21 \mathrm{Zu}$ diesem Begriff s. die Klarstellung in Erwägungsgrund 6 der Richtlinie 2000/43/EG: »Die Europäische Union weist Theorien, mit denen versucht wird, die Existenz verschiedener menschlicher Rassen zu belegen, zurück. Die Verwendung des Begriffs >Rasse` in dieser Richtlinie impliziert nicht die Akzeptanz solcher Theorien."

22 Beispiel: OLG Köln NJW 2010, 1676, 1677 f. (Anspruch aus § 831 Abs. 1S. 1 BGB bejahend).

23 S. dazu MünchKomm-BGB/Thüsing, 8. Aufl. 2018, §19 AGG Rn. 139.

24 Erman/Armbrüster, BGB, 16. Aufl. 2020, § 19 AGG Rn. 11. 
verträgen erschweren oder verwehren und sie dadurch ausgrenzen sowie in ihrer Persönlichkeit herabwürdigen. Der Nachfrager wird hingegen durch das Benachteiligungsverbot nicht daran gehindert, z. B. Läden oder Restaurants zu meiden, die von Angehörigen einer bestimmten Ethnie oder Glaubensgemeinschaft geführt werden. Äußert er sich zugleich abschätzig, so kann dies im Hinblick auf die Ehrdelikte der $\S \S 185 \mathrm{ff}$. StGB strafrechtlich bedeutsam sein; hingegen wird seine negative Vertragsabschlussfreiheit (also die durch Art. 2 Abs. 1 GG geschützte Freiheit, einen Vertrag nicht abzuschließen) durch das AGG nicht eingeschränkt.

Variante 2: Die Agentur sucht im Internet für die Aufgabe, als Weihnachtsmann aufzutreten, ausschließlich männliche Bewerber, oder sie lässt bei der Stellenvergabe nur sie zum Zuge kommen. In solchen Fällen kommt auf Seiten der Agentur im Verhältnis zu Bewerberinnen und Bewerbern um die Tätigkeit als Weihnachtsmann ein Verstoß gegen das geschlechtsbezogene Benachteiligungsverbot in Betracht. Geht es um eine Beschäftigung als freie Mitarbeiter/in, so liegt darin tatbestandlich ein Verstoß gegen $\S 19$ Abs. 1 Nr. 1 AGG. Gerechtfertigt ist eine Ungleichbehandlung wegen des Geschlechts dann, wenn ein sachlicher Grund vorliegt (§ 20 Abs. 1 S. 1 AGG). Ein solcher Grund kann etwa darin liegen, dass zu bestimmten Sportveranstaltungen nur Frauen oder nur Männer zugelassen werden. Die Tätigkeit als Weihnachtsmann kann indessen, wie dargelegt, angesichts der ohnehin zum vertragsgemäßen Erscheinungsbild gehörenden Verkleidung grundsätzlich auch von Frauen ausgeübt werden. Es ist daher nicht gerechtfertigt, wenn sie von vornherein rundheraus und ohne Prüfung ihrer individuellen Eignung aus dem Bewerberkreis ausgeschlossen werden. Werden Personen als Weihnachtsmann eingesetzt, die nicht freiberuflich, sondern als Beschäftigte i.S.v. § 6 Abs. 1 AGG (insbesondere: Arbeitnehmer/innen) tätig sind, gilt anstelle des Benachteiligungsverbots nach § 19 AGG dasjenige des $\S 7$ AGG. In diesem Fall greift statt $§ 20$ AGG der Rechtfertigungstatbestand des $\S 8$ Abs. 1 AGG ein. Demnach ist eine unterschiedliche Behandlung (u.a.) wegen des Geschlechts zulässig, wenn es sich wegen der Art der Tätigkeit oder der Bedingungen ihrer Ausübung um eine wesentliche und entscheidende berufliche Anforderung handelt. Dies lässt sich, wie dargelegt, bei der Tätigkeit als Weihnachtsmann nicht ohne Weiteres bejahen. Hingegen wäre es etwa gerechtfertigt, wenn eine Agentur, die Models für Damenmodeschauen beschäftigt, in ihrer Stellenausschreibung ausschließlich Frauen sucht.

\section{Akt: Das Weihnachtsgeschenk}

Anna ist mittlerweile mit der Weihnachtsfrau in einen hitzigen Disput geraten. Es geht um die Rolle der Frau in der modernen Gesellschaft. Anna hält der Weihnachtsfrau vor, dass Frauen nicht in jeder Hinsicht den Männern nacheifern und deren traditionelle Berufsfelder erobern sollten. Thomas ist es unangenehm, wie heftig Anna ihre Position vorbringt. Er findet die Weihnachtsfrau durchaus sympathisch und möchte sie etwas aufmuntern. Spontan überreicht er ihr daher das hübsch verpackte Geschenk, das er schon mittags mit einem Aufkleber $»$ Für meine Liebste« für die - wie zwischen Anna und Thomas üblich erst nach dem Essen geplante Bescherung unter den Weihnachtsbaum gelegt hatte. Anna hatte den Zettel schon erblickt und war gespannt darauf, was Thomas ihr wohl zugedacht hat. Jetzt ist sie empört. In scharfem Tonfall fordert sie die Weihnachtsfrau auf, ihr sofort das Päckchen zurückzugeben, da es ihr gehöre. Die Weihnachtsfrau entgegnet nur: »Geschenkt ist geschenkt!« und geht daran, die kunstvolle Verpackung zu öffnen. Wer hat recht?

Bevor die Frage beantwortet wird, lohnt ein Ausflug ins Schenkungsrecht. Dieses Rechtsgebiet birgt einige Fallstricke. Sie hängen insbesondere damit zusammen, dass der Gesetzgeber den Schenker davor bewahren will übereilt rechtsgeschäftliche Bindungen einzugehen, die er womöglich später bereut. $\mathrm{Zu}$ unterscheiden sind die sog. Handschenkung gem. § 516 BGB und das Schenkungsversprechen gem. § 518 BGB. Bei der Handschenkung erfolgt die Zuwendung als das Erfüllungsgeschäft zugleich (uno actu) mit dem Abschluss des schuldrechtlichen Verpflichtungsgeschäfts. In diesem Fall ist der Schenker allein schon durch den sofortigen Vollzug hinreichend gewarnt: Wer sehenden Auges eigene Vermögensgegenstände aus der Hand gibt, weiß, was er tut. Er kann sich auf diese Weise rechtswirksam nahezu seines gesamten Vermögens begeben, sofern nicht eine arglistige Täuschung oder eine Drohung (§ 123 BGB), Sittenwidrigkeit ( 138 BGB), eine Benachteiligung der Gläubiger des Schenkers (vgl. die Regelungen des AnfG) oder ein sonstiger Tatbestand vorliegt, welcher der privatautonomen Gestaltung Grenzen setzt. Handelt es sich bei dem Schenkungsgegenstand um ein Grundstück, so bewirkt das Erfordernis notarieller Beurkundung des Schenkungsvertrags nach $\S 311 \mathrm{~b}$ BGB einen Schutz vor Übereilung. Zudem steht dem Schenker nach $\S 528$ Abs. 1 BGB ein Rückforderungsanspruch wegen Verarmung zu, auf den ggf. der Sozialhilfeträger zugreifen kann (vgl. § 93 SGB XII). Nicht zuletzt bietet § 530 BGB dem Schenker ein Widerrufsrecht wegen groben Undanks, der etwa in einem tätlichen Angriff des Beschenkten liegen kann. Jenseits dieser Ausnahmefälle gilt aller- 
dings bei einer wirksam erfolgten Handschenkung in der Tat: »Geschenkt ist geschenkt«.

Bei einem Schenkungsversprechen wird der Schenker durch ein schenkungsrechtliches Formerfordernis vor unbedachter Freigiebigkeit geschützt: Das Versprechen bedarf gem. § 518 Abs. 1 S. 1 BGB der notariellen Beurkundung; anderenfalls ist es nach $\S 125$ S. 1 BGB nichtig. Die notarielle Beurkundung ist die strengste Form, die das BGB für Rechtsgeschäfte kennt. Hierbei hat der Notar - anders als bei einer notariellen Unterschriftsbeglaubigung nicht lediglich die Identität desjenigen zu bestätigen, der die Unterschrift geleistet hat. Vielmehr muss der Notar den gesamten Vertragstext in Anwesenheit der Beteiligten verlesen; überdies trifft ihn eine weit reichende Belehrungspflicht ( $\$ 17$ BeurkG). Eine gewisse Schutzlücke besteht freilich insofern, als der Schenker einen Vertreter entsenden kann; in diesem Fall bedarf zwar das vom Vertreter geschlossene Geschäft der notariellen Beurkundung, nicht aber nach der - deshalb rechtspolitisch umstrittenen $-^{25}$ Vorschrift des § 167 Abs. 2 BGB die Vollmachtserteilung durch den Schenker. Allerdings kommt bei dem Formerfordernis nach $\S 518$ Abs. 1 S. 1 BGB ebenso wie bei demjenigen nach $\S 311 \mathrm{~b}$ BGB eine teleologische Reduktion des $\S 167$ Abs. 2 BGB in Betracht, wenn bereits die Erteilung der Vollmacht zu einer Bindung des Vertretenen führt. ${ }^{26}$

Ein Verstoß gegen das Formerfordernis wird gem. $\S 518$ Abs. 2 BGB dadurch geheilt, dass die schenkweise versprochene Leistung tatsächlich bewirkt wird. Der Vollzug des Schenkungsversprechens führt nämlich - wie bei der Handschenkung, nur eben nicht zugleich mit dem Verpflichtungsgeschäft - dem Schenker vor Augen, dass er einen Vermögensgegenstand weggibt. Er bedarf dann nicht mehr des Schutzes vor Übereilung, den das Formerfordernis bei der Schenkung als einseitig verpflichtendem Vertrag dem Schenker dann bieten soll, wenn der Vollzug erst für die Zukunft versprochen wird.

Inwieweit sind diese Regeln für den Streit zwischen Anna und der Weihnachtsfrau von Interesse? Anna macht hier die Vindikation geltend, also den Anspruch auf Herausgabe des Eigentums gem. §985 BGB. Ursprünglich war Thomas Eigentümer des Geschenks. Es kommt in Betracht, dass er sein Eigentum bereits dadurch an Anna verloren hat, dass er das Päckchen unter den Weihnachtsbaum gelegt hat. Der Übergang des Eigentums richtet sich grundsätzlich nach § 929 S. 1 BGB. Hierbei ist strikt zwischen Verpflichtungs- und Verfügungsgeschäft zu unter-

25 S. dazu Staudinger/Schilken, BGB, 2019, § 167 Rn. 20.

26 MünchKomm-BGB/Schubert, 8. Aufl. 2018, § 167 Rn. 20, 31; für fallgruppenunabhängige teleologische Reduktion Staudinger/Schilken, BGB, 2019, § 167 Rn. 20. scheiden (Trennungsprinzip). Zudem gilt es wegen des Abstraktionsprinzips - das bisweilen mit dem Trennungsprinzip verwechselt wird - die Wirksamkeit des einen von der Wirksamkeit des anderen Geschäftes gesondert zu beurteilen. ${ }^{27}$ Eine Ausnahme von diesen Prinzipien - wie sie im Hinblick auf das "Durchschlagen" der Sittenwidrigkeit eines Verpflichtungsgeschäfts auf das Verfügungsgeschäft diskutiert wird $-^{28}$ kommt hier nicht in Betracht.

Für die Eigentumsverhältnisse ist allein das Verfügungsgeschäft maßgeblich. Gleichwohl kann es für das Verständnis hilfreich sein, den obigen Exkurs zum Schenkungsrecht im Blick zu behalten. Der für Anna erkennbarer Wille von Thomas war auf eine Handschenkung gerichtet; er ging also dahin, dass (erst) im Zeitpunkt der Bescherung eine Zuwendung i.S.v. § 516 Abs. 1 BGB erfolgen sollte. Es hat also mangels Einigung und Übergabe noch kein Eigentumsübergang an Anna stattgefunden. Wollte man stattdessen bereits in der Ankündigung, Anna ein Geschenk machen $\mathrm{zu}$ wollen, ein (von ihr konkludent angenommenes) Schenkungsversprechen erblicken, so wäre dieses beurkundungsbedürftig und der Formmangel noch nicht durch die tatsächliche Leistungsbewirkung geheilt. Daher würde sich insoweit auf der hier interessierenden sachenrechtlichen Ebene nichts anderes als bei einer Handschenkung ergeben. Mithin ist Anna nicht Eigentümerin des Geschenks geworden, so dass die von ihr geltend gemachte Vindikation gem. § 985 BGB entfällt. Vielmehr hatte die Weihnachtsfrau durch Übergabe den Besitz erlangt, und sie hat ihn weiterhin inne. Die nach § 929 BGB zudem erforderliche Einigung ist zwischen Thomas und der Weihnachtsfrau konkludent zustande gekommen, als er ihr das Päckchen aushändigte. Als Eigentümer war Thomas grundsätzlich auch zu der Verfügung berechtigt. Daher ist die Weihnachtsfrau Eigentümerin des Geschenks geworden. Im Folgenden soll der Sachverhalt noch in verschiedener Hinsicht variiert werden.

Variante 1: Wem würde das Geschenk gehören, wenn Thomas es bereits Anna ausgehändigt hätte, mit der Maßgabe, dass es ihr mit der Bescherung gehören solle, die Thomas aber sodann wegen des Streits »abbläst«? Die Übergabe läge dann vor. Bei der Einschränkung der Einigung hinsichtlich der Bescherung kann es sich um eine Befristung gem. § 163 BGB oder um eine aufschiebende Bedingung gem. §158 Abs. 1 BGB handeln. Eine Verfügung kann - anders als einseitige Rechtsgeschäfte, insbesondere die Ausübung eines Gestaltungsrechts - grund-

27 Instruktiv Lieder/Berneith, JuS 2016, 673, $674 \mathrm{f}$.

28 Vgl. dazu MünchKomm-BGB/Armbrüster, 8. Aufl. 2018, §138 Rn. 165. 
sätzlich unter einer Bedingung oder Befristung erfolgen. Welcher dieser Fälle hier anzunehmen ist, hängt davon ab, ob man die Bescherung als gewisses oder ungewisses Ereignis ansieht. Für ein gewisses Ereignis und somit für eine Befristung spricht es, wenn das Eintreten des Ereignisses nur noch eine Frage der Zeit ist. ${ }^{29}$ Dies ist insbesondere bei einem Kalenderdatum der Fall. Dagegen wird ein ungewisses Ereignis dadurch charakterisiert, dass bereits zweifelhaft ist, ob es überhaupt eintreten wird. ${ }^{30}$ Maßgeblich für die Abgrenzung ist der Parteiwille; dabei legt eine nur kurze Zeitspanne eine Befristung nahe. Hier spricht der Umstand, dass die Bescherung bereits fest eingeplant und verabredet war, für ein aus Sicht der Vertragschließenden ex ante gewisses Ereignis und damit für eine Befristung. Diese ist Bestandteil der Willenserklärung, also des Angebots i.S.v. § 145 BGB zum Eigentumserwerb. Die Befristung bedurfte damit gem. § 147 BGB einer zumindest konkludenten Annahme durch Anna. Davon, dass diese vorliegt, ist bei lebensnaher Betrachtung auszugehen. Ein Zugang der Annahmeerklärung - nicht diese Erklärung selbst - ist hier nach der Verkehrssitte gem. § 151 BGB entbehrlich. ${ }^{31}$

Klärungsbedürftig ist, ob der Umstand, dass Thomas die Bescherung anschließend »abgeblasen« hat, einem Eigentumserwerb durch Anna entgegensteht. Dies ist nicht der Fall, wenn $\S 162$ Abs. 1 BGB anwendbar und erfüllt ist. Nach dieser Vorschrift gilt eine Bedingung als eingetreten, wenn derjenige, $\mathrm{zu}$ dessen Nachteil sie gereichen würde, treuwidrig ihren Eintritt verhindert. Just diese Norm zählt allerdings (neben $\S 159$ BGB) nicht zu den nach § 163 BGB auf die Befristung entsprechend anwendbaren Vorschriften des Bedingungsrechts. Es kommt jedoch in Betracht, denselben Rechtsgedanken über § 242 BGB heranzuziehen. Dafür spricht, dass beide Modifikationen des Vertrags eng verwandt sind und es sich bei § 162 BGB lediglich um eine Konkretisierung des allgemeinen Grundsatzes von Treu und Glauben handelt. ${ }^{32}$ Indem Thomas die Bescherung absagte, hat er den Eintritt des Fristbeginns verhindert. Das geschah auch in treuwidriger Weise, da er damit den Erfolg der von ihm bereits zuvor getroffenen Verfügung vereiteln wollte. Seine Manipulation führt gem.

29 Staudinger/Bork, BGB, 2015, § 163 Rn. 4.

30 BGH NJW-RR 2016, 842 Rn. 19; BeckOK-BGB/Rövekamp, 55. Ed., 1. $8.2020, \S 158$ Rn. 3.

31 Vgl. BGH NJW 2004, 287, 288; OLG Brandenburg NJW-RR 2018, 530 Rn. 28; Medicus/Petersen, Bürgerliches Recht, 27. Aufl. 2019, Rn. 55.

32 Erman/Armbrüster, 16. Aufl. 2020, § 163 Rn. 3; für analoge Anwendung von § 162 BGB (mit gleichem Ergebnis) etwa Jauernig/Mansel, BGB, 17. Aufl. 2018, § 163 Rn. 1; MünchKomm-BGB/Westermann, 8. Aufl. 2018, § 163 Rn. 6.
$\S 242$ BGB dazu, dass die Bescherung fingiert wird. Mithin hat Anna zu dem Zeitpunkt, zu dem die Bescherung geplant war, Eigentum erlangt.

Variante 2: Ergibt sich ein Unterschied zu Variante 1, wenn Thomas das Geschenk noch nicht an Anna übergeben, sondern sich mit ihr lediglich darauf verständigt hat, dass es ihr mit der Bescherung gehören soll, er es aber dann - wie im Ausgangsfall - an die Weihnachtsfrau übergibt? Allein die befristete Einigung mit Anna stellt noch keine Verfügung i.S.v. § 161 Abs. 1 BGB dar, solange die für die Übereignung gem. §929 BGB erforderliche Übergabe fehlt. Bis zur Übergabe kann Thomas daher sein Angebot auf Übereignung an Anna frei widerrufen. ${ }^{33}$ Das hat er hier jedenfalls konkludent getan, indem er das Geschenk der Weihnachtsfrau aushändigte. Letztere ist damit auch in Variante 2 Eigentümerin geworden; sie muss das Geschenk nicht an Anna herausgeben. Wegen der freien Widerrufbarkeit des Übereignungsangebots wäre Anna im Übrigen auch dann nicht vor Zwischenverfügungen geschützt gewesen, wenn die Einigung mit Thomas nicht befristet oder bedingt, sondern sofort wirksam gewesen wäre, solange nur die Übergabe an Anna noch ausstand.

Variante 3: Wie Variante 2; Anna kommt aber Thomas zuvor und nimmt das Päckchen an sich. Hat sie dadurch Besitz daran erlangt? Der Übergang des Besitzes an einer beweglichen Sache richtet sich nach den $\$ \S 854 \mathrm{ff}$. BGB. Für die Besitzerlangung genügt es gem. § 854 Abs. 1 BGB grundsätzlich, dass die tatsächliche Sachherrschaft an der beweglichen Sache ausgeübt wird und dass dies von einem Besitzwillen (Beherrschungswillen) getragen ist. Hingegen reicht es nicht, wenn lediglich eine tatsächliche $\mathrm{Zu}$ griffsmöglichkeit besteht. Eine Besitzerlangung i.S.v. $\S 854$ Abs. 1 BGB kann auch durch einfaches Ergreifen erfolgen. ${ }^{34}$ Es genügt also, wenn jemand das unter dem Weihnachtsbaum befindliche Geschenk an sich nimmt. Dadurch verliert der bisherige Besitzer automatisch den unmittelbaren Besitz an der Sache. ${ }^{35}$

Für die Besitzerlangung ist nach überwiegender Auffassung ${ }^{36}$ eine gewisse zeitliche Dauer der Sachbeziehung nötig, wobei die Umstände des Einzelfalles zu beachten sind. Nach der Gegenansicht ${ }^{37}$ genügt für das Ausüben ei-

33 S. nur BGH 1979, 213, 214; BeckOK-BGB/Kindl, 55. Ed., 1. 8. 2020, $\S 929$ Rn. 18.

34 BGHZ 27, 360 = NJW 1958, 1286; MünchKomm-BGB/Schäfer, 8. Aufl. 2020, § 854 Rn. 46; jurisPK-BGB/Gies, 9. Aufl. 2020, § 854 Rn. 32. 35 Lüke, Sachenrecht, 4. Aufl. 2018, Rn. 87.

36 St. Rspr., s. nur BGH NJW-RR 2017, 818 Rn. 18; F. Ebert, NJW 2016, 3206, 3208.

37 MünchKomm-BGB/Schäfer, 8. Aufl. 2020, § 854 Rn. 30; vertiefend Hartung, Besitz und Sachherrschaft, 2001, S. $130 \mathrm{ff}$. 
ner tatsächlichen Sachherrschaft schon ein sog. Kurzbesitz, also etwa das kurzzeitige Ergreifen, da diese Sachherrschaft als Realakt nur einen natürlichen Willen erfordert. Für diese Ansicht spricht, dass dem richtigen Besitzer demnach gegen den fehlerhaften Besitzer gem. §§ $858 \mathrm{ff}$. BGB genügend Schutzrechte zur Verfügung stehen. Zudem ist die Dauer des Besitzes ein ungenaues Kriterium zur Feststellung eines Besitzerwerbs. Es genügt daher, wenn die Beziehung des Besitzanwärters zu der Sache sich wenn auch nur für kurze Zeit - verfestigt hat. Dafür ist auch bedeutsam, in welcher Sphäre der potentielle Besitzerwerber mit der Sache in Kontakt kommt. ${ }^{38}$ Der Destinatär erlangt demnach schon durch das Ergreifen des Geschenks den Besitz daran; bei einer Zusendung per Post wird der Besitz bereits mit dem Erhalt des Pakets verschafft. Eine abweichende Beurteilung kommt etwa dann in Betracht, wenn der Destinatär das Geschenk in den Räumlichkeiten des Schenkenden unter dessen Augen an sich nimmt. In diesem Fall kann die tatsächliche Sachherrschaft des Schenkenden fortwirken, so dass der für den Besitzwechsel erforderliche vollständige Verlust des Besitzes auf Seiten des bisherigen Besitzers fehlt. Hier könnte man angesichts des von Thomas und Anna gemeinsam bewohnten Hauses trefflich darüber streiten, ob die Voraussetzungen für diese Ausnahme erfüllt sind.

Auch wenn Anna in der Variante 3 tatsächlich Besitz erlangt haben sollte, muss allerdings noch geklärt werden, ob dies auf Veranlassung von Thomas erfolgte, was Voraussetzung für eine Übergabe i.S.v. § 929 S. 1 BGB wäre. ${ }^{39}$ Spätestens dies muss verneint werden, da der Zugriff von Anna auf das Geschenk, das sie nach dem Willen von Thomas erst nach der Bescherung erhalten sollte, eine verbotene Eigenmacht darstellt, welche den Eigentumserwerb nicht begründen kann..$^{40}$

Am Rande vermerkt: Die Aufschrift »für meine Liebste« mag auf einem Geschenk unterm Weihnachtsbaum nur selten Verwechslungen oder gar Eifersuchtsdramen auslösen. In einer Verfügung von Todes wegen (Testament, Erbvertrag) ist es hingegen generell keine gute Idee, die zu bedenkende Person nicht namentlich, sondern nur mit einem Superlativ (Beispiel: "meinem Liebsten«) oder unspezifischen Kosenamen (Beispiel: »meinem Schatz«) zu benennen. Dann gelten nämlich gem. § 2073 BGB alle auf die Bezeichnung passenden Personen als zu gleichen

38 BeckOGK/Götz, 1. 7. 2020, § 854 Rn. 74; BeckOK-BGB/Fritzsche, 55. Ed., 1. 8. 2020, § 854 Rn. 23.

39 BGH NJW 1996, 2654, 2655; BeckOGK/Klinck, 1. 7. 2020, § 929

Rn. 73; Hk-BGB/Schulte-Nölke, 10. Aufl. 2019, § 929 Rn. 10.

40 RGZ 137, 23 = BeckRS 1932, 100289 Rn. 5; MünchKomm-BGB/

Oechsler, 8. Aufl. 2020, § 929 Rn. 49.
Teilen bedacht; steht hingegen fest, dass der Erblasser nur eine einzige Person bedenken wollte, ist die Einsetzung unwirksam. ${ }^{41}$ Es kommt auch vor, dass eine beim Verfassen der Verfügung von Todes wegen völlig eindeutige Einsetzung später mehrdeutig wird (Beispiel: "meine Ehefrau « bei späterer Scheidung und Wiederheirat). Dann ist die Verfügung nach der dispositiven Auslegungsregel des $\S 2077$ Abs. 1 S. 1 BGB im Zweifel unwirksam.

\section{Akt: Das unverhoffte Verlobungsgeschenk}

$\mathrm{Zu}$ guter Letzt erfährt der Heiligabend unverhofft - wenn auch nur kurzzeitig - eine romantische Wendung: Noch während sie mit Anna streitet, packt die Weihnachtsfrau das Geschenk aus. Es enthält einen silbern funkelnden Verlobungsring! Thomas zwinkert ihr lächelnd zu, denn er sieht in der Situation eine willkommene Gelegenheit Anna spielerisch zu zeigen, dass er auch bei anderen Frauen eine Chance hätte. Der Weihnachtsfrau wiederum fällt jetzt schlagartig wieder ein, dass sie unlängst auf den Weihnachtsmarkt bei einer Wahrsagerin war. Diese hatte ihr offenbart, dass sich ihr Leben an Heiligabend grundlegend ändern würde. Jetzt ist der Weihnachtsfrau klar, was die Wahrsagerin gemeint hatte. Sie fällt Thomas spontan um den Hals und sagt nur: »Ich bin so glücklich!«. Diese Äußerung kann in mehrerlei Hinsicht ein Fehler gewesen sein. Wieso?

Die Äußerung der Weihnachtsfrau lässt sich als Annahme eines Angebots von Thomas begreifen, ein Verlöbnis mit ihm einzugehen. Dies wäre schon deshalb ein Fehler, weil Thomas - so muss es ihr jedenfalls erscheinen offenbar in seiner Lebensplanung recht unstet ist. Vor allem aber gefährdet die Weihnachtsfrau durch die Erklärung ihr Geschenk, genauer: sie setzt sich einem Rückforderungsanspruch von Thomas aus. Für Verlobte hält $\S 1301$ S. 1 BGB nämlich in Abweichung von den allgemeinen Vorschriften über den Widerruf einer Schenkung eine Sonderregel bereit: Kommt es nicht zur Eheschließung, so kann jeder Verlobte von dem anderen die Gegenstände herausverlangen, die er ihm "geschenkt oder zum Zeichen des Verlöbnisses gegeben hat«. Es handelt sich um einen Bereicherungsanspruch wegen Zweckverfehlung gem. $\S 812$ Abs. 1 S. 2 Fall 2 BGB (condictio ob rem). Der Anspruch setzt voraus, dass zum Zeitpunkt der Schenkung ein wirksames Eheversprechen bestand. Darunter versteht man einen Vertrag, durch den sich zwei Personen gegen-

41 BGH WM 1975, $737 \mathrm{f}$. 
seitig versprechen, die Ehe miteinander einzugehen..$^{42}$ Dieser Vertrag bedarf keiner Form; er kann auch durch konkludentes Verhalten zustande kommen. Ob man hier allerdings davon auszugehen hat, dass Thomas der Weihnachtsfrau einen entsprechenden Antrag gemacht hat, ist zweifelhaft. Allein die Hingabe eines ursprünglich für eine andere Person bestimmten Verlobungsrings hat nicht ohne Weiteres einen auf eine Verlobung gerichteten Erklärungswert. Die Umstände - die nur sehr flüchtige Bekanntschaft der beiden, der vorangegangene Streit mit Anna und die dadurch aufgeheizte Stimmung, das Augenzwinkern - sprechen gegen einen solchen Willen. Jedenfalls aber wäre die Erklärung nach § 118 BGB nichtig, da sie in der Erwartung abgegeben wurde, der Mangel an Ernstlichkeit werde nicht verkannt.

Die Weihnachtsfrau hat also noch einmal Glück gehabt. Die Zuwendung als solche hatte Thomas nämlich gewollt; sie ist nicht im Hinblick auf eine Verlobung mit der Weihnachtsfrau erfolgt, sondern zu deren Trost für den Streit mit Anna. Thomas könnte den Ring daher nicht nach $\S 1301$ BGB, sondern allein nach den Regeln des Schenkungsrechts zurückfordern. Für eine Verarmung von Thomas i.S.v. § 528 BGB sind keinerlei Anhaltspunkte ersichtlich. Auch ein Widerruf der Schenkung wegen groben Undanks ( $\$ 530$ BGB) kommt nach dem Sachverhalt nicht in Betracht. Die Weihnachtsfrau kann jetzt also mit dem Verlobungsring, wenn auch ohne dazugehörigen Verlobten, die Bühne des Reihenhauses verlassen.

\section{Epilog (Nachrede)}

Zum Glück verläuft nicht jeder Heiligabend so turbulent, wie es bei den Beteiligten unseres Kurzdramas der Fall war. Allen Leserinnen und Lesern des Beitrags sei hiermit ein friedvoller Verlauf gewünscht. Dafür, dass dieser Wunsch in Erfüllung geht und man nicht selbst Rechtsfälle produziert, lassen sich aus den angesprochenen Themen einige praktische Hinweise entnehmen. Am trivialsten ist wohl derjenige, dass es nicht ratsam ist, Töpfe mit Öl unbeaufsichtigt auf dem Herd stehen zu lassen. Dasselbe gilt, auf die wärmere Jahreszeit bezogen, für die Praxis, brennbare Flüssigkeiten in die offene Flamme eines Grills zu schütten. Beides kommt in der Lebenswirklichkeit immer wieder vor und führt zu unschönen Haftungs- und Versicherungsfällen.

Eine weitere Lehre betrifft die Bescherung: Wer als Schenker sichergehen will, sollte die Geschenke erst dadurch der zu bedenkenden Person zuordnen, dass er sie ihr übergibt. Wird die Zuordnung schon vor der gewollten Besitzverschaffung offengelegt, so kann dies bei ungeduldigen Destinatären - wie in Akt 4 gesehen - für Verwicklungen sorgen. Und nicht zuletzt ist der traditionelle Brauch, den Heiligabend für Eheversprechen zu nutzen, für die an diesem Abend Beschenkten riskant. Erweist sich der oder die Auserwählte im grauen Januarlicht doch nicht als die perfekte Wahl, so müssen die am Heiligabend überreichten Präsente dann, wenn sie als Verlobungsgeschenke einzuordnen sind, allesamt wieder herausgegeben werden. Wer sich also gern beschenken lässt und es mit dem Verlöbnis nicht ganz so eilig hat, sollte sicherheitshalber mit diesem Ansinnen bis zum Silvestertag - der übrigens ebenso wie der Dreikönigstag nicht zu den neun bundeseinheitlich geregelten Feiertagen zählt, sondern nur in einigen Bundesländern Feiertag ist - warten.

42 BGHZ 28, 376, 377 = NJW 1959, 1032 (Ls.). 